\title{
Fachkonzept mit Widersprüchen
}

\section{»Kundenforderungen « und die Notwendigkeiten der Praxis}

\author{
Wolfgang Trunk
}

Die Bundesagentur für Arbeit hat einige Anforderungen an die Werkstätten für bebinderte Menschen neu geordnet. Durch das »Fachkonzept « sollen die Möglichkeiten zur selbstbestimmten Teilhabe behinderter Menschen am Arbeitsleben verbessert und somit ein Beitrag zur Umsetzung der in der VN-Konvention über die Rechte behinderter Menschen verankerten Zielsetzung beruflicher Inklusion geleistet werden. Das Konzept enthält jedoch allerlei Widersprüche, wie der Autor des nachfolgenden Beitrags meint.

Der Umgang mit Widersprüchen zählt seit jeher zur Professionalität Sozialer Arbeit. Soziale Hilfen gehen immer von Problemlagen aus, also von Erfordernissen und Gegebenheiten, die nicht zueinander passen. Allerdings können sich die Akteure den jeweiligen Typ von Widerspruch nicht aussuchen.

Hat man Glück, dann bekommt man es mit einem produktiven Widerspruch zu tun, der dem Klienten Gelegenheit gibt, seine Handlungsfähigkeit zu erweitern, seine Lebensqualität zu optimieren und seine Persönlichkeit zu entwickeln. Wenn sie auch Kraft kostet, so ergibt die gemeinsame Arbeit an solchen Widersprüchen doch einen erfahrbaren Sinn. Häufig ist man auch mit Widersprüchen konfrontiert, die nicht aus der Persönlichkeit des Klienten resultieren, sondern aus dem Spannungsverhältnis zwischen den Notwendigkeiten der Hilfe einerseits und ungünstigen Rahmenbedingungen andererseits. Hier ringt der professionelle Helfer mehr mit Vertretern von Behörden und Gesellschaft als mit dem Klienten.

\footnotetext{
Wolfgang Trunk ist Mitarbeiter des Frankfurter Vereins für soziale Heimstätten. E-Mail wolfgang.trunk@frankfurterverein.de
}

Immerhin - auch dieser Kampf ist repräsentativ, und er kann dazu beitragen, dass sich der Klient am eigenen Beispiel der sozialen Wirklichkeit bewusst wird, in der er lebt.

Solange ein Kampf um die Lebensqualität stattfindet, ist jedenfalls eine Bewegung vorhanden, die aus Widersprüchen resultiert, und die im günstigen Fall zu einer Entwicklung führt. Ganz anders stellen sich die Dinge dar, wenn gar keine Widersprüche zu erkennen sind. Diese Variante wird traditionell von den Institutionen des Bildungswesens favorisiert; sie geben die Lernwege für alle vor, und wer ihnen willig folgt, der werde keine Probleme bekommen - und wenn doch, dann liege das aber nicht an der Institution.

Leider färbt das Beispiel des Bildungswesens ab: Zunehmend beanspruchen auch die Sozialleistungsträger in Deutschland zu wissen, welche Wege die Klienten gehen sollen, und wie soziale Einrichtungen dementsprechend ihre Arbeit zu machen haben.

Ein jüngeres Beispiel dafür sind die Kundenforderungen der Arbeitsagentur für die berufliche Bildung in den Werkstätten für Behinderte, die im Herbst 2010 in Kraft getreten sind. Aus wissenschaftlicher Sicht sind diese Forderungen schlichtweg obsolet; sie firmieren gleichwohl als »Fachkonzept «" und sollen als eine Art Meta-Konzeption für die berufliche Bildung behandelt werden. So widerspruchsfrei das Papier daherkommt, so erfahrbar werden die Widersprüche, wenn man versucht, die bürokratischen Vorgaben der Arbeitsagentur auf die reale Praxis zu beziehen.

Erster Widerspruch: Die Arbeitsagentur fordert, dass sich die berufliche Bildung an den »Fähigkeiten der behinderten Menschen « orientiert (Punkt 1.). Das kann im Einklang mit dem Prinzip stehen, bei allen Maßnahmen der Fähigkeitsentwicklung vom biographischen
Standort des Mitarbeiters auszugehen; es bleibt jedoch offen, ob hier die umfassende Weiterführung einer persönlichen Entwicklung gemeint ist, oder ob solche Einzelfähigkeiten in den Fokus gerückt werden, die am ehesten eine ökonomische Verwertbarkeit erwarten lassen.

Zweiter Widerspruch: Die Arbeitsagentur fordert, dass sich die berufliche Bildung in den Werkstätten "an den Interessen ... der behinderten Menschen« orientiert (Punkt 1.). Das kann im Einklang mit dem Prinzip stehen, bei allen Maßnahmen der Fähigkeitsentwicklung die Interessenlage der Person zu beachten; es bleibt jedoch offen, ob hier die objektiven, grundlegenden und längerfristigen Lebensinteressen im Mittelpunkt stehen oder ob aktuelle Neigungen und Wunschvorstellungen handlungsleitend werden sollen, die mit dem Interesse des Leistungsträgers korrespondieren.

Dritter Widerspruch: Das Papier der Arbeitsagentur bezeichnet das »Erlernen von Fähigkeiten und Fertigkeiten « zum "Ausführen von beruflichen Tätigkeiten « als Zweck der Bildungsarbeit in den Werkstätten (Punkt 5.1). Das kann im Einklang mit dem Prinzip stehen, die Arbeit als zentralen Lerngegenstand zu nutzen; es bleibt jedoch offen, ob hier die realen und konkreten Arbeitsaufgaben in einer Werkstatt gemeint sind, oder ob an deren Stelle die Kunstprodukte von didaktisch aufbereiteten »Maßnahmen « treten sollen.

Vierter Widerspruch: Die Arbeitsagentur fordert, dass man die behinderten Mitarbeiter anleitet, »ihr Leben eigenverantwortlich $\mathrm{zu}$ planen und $\mathrm{zu}$ führen « (Punkt 3.3.). Das kann im Einklang mit dem Prinzip stehen, die behinderten Personen als die Subjekte ihrer Fähigkeitsentwicklung zu behandeln. Es bleibt jedoch offen, wie der Leistungsträger mit dem Umstand ver- 
fahren will, dass die Subjektfunktionen einer Person von anderen zwar begünstigt, nicht aber produziert oder garantiert werden können.

Fünfter Widerspruch: Die Arbeitsagentur fordert, dass die behinderten Mitarbeiter lernen sollen, »ihr Leben eigenverantwortlich ... zu führen« (Punkt 3.3.). Das kann im Einklang mit dem Prinzip stehen, dass die Personen ihre Lerngründe aus der erfahrbaren Erweiterung ihrer Handlungsfähigkeit gewinnen. Es bleibt jedoch offen, ob sich die Mitarbeiter auf die üblichen und vorgefundenen Handlungsmöglichkeiten beschränken sollen oder ob die Erweiterung der Handlungsfähigkeit auch einschließt, dass die Mitarbeiter selbst Einfluss auf ihre Lebens- und Arbeitsbedingungen nehmen können, um ihre Handlungsmöglichkeiten zu erweitern.

Sechster Widerspruch: Die Arbeitsagentur fordert, dass die Werkstätten Anwesenheitslisten führen, in denen »unterweisungsfreie Zeiten und Fehlzeiten zu kennzeichnen " sind (Punkt 3.5.). Das widerspricht dem Prinzip, ein Lernklima zu gestalten, das von Fremdkontrolle und Abwehrhaltungen möglichst frei ist.

Siebter Widerspruch: Die Arbeitsagentur spricht von einem »Unterstützungsbedarf « und fordert, dass jedem behinderten Mitarbeiter eine bestimmte Person als »Bildungsbegleiter « zugeordnet wird (Punkt 3.2.2.). Das kann im Einklang mit dem Prinzip stehen, die behinderten Mitarbeiter auf ihren Lernwegen individuell zu unterstützen; es bleibt jedoch offen, auf welche zusätzlichen personellen Ressourcen die Werkstätten zugreifen sollen, um eine andragogisch kompetente Begleitung zu gewährleisten.

Achter Widerspruch: Die Arbeitsagentur fordert, dass die berufliche Bildung »fördernd und fordernd zu planen und durchzuführen « ist (Punkt 5.). Das kann im Einklang mit dem Prinzip stehen, mit der individuellen Bildung an den Problemen anzusetzen, auf die ein Mitarbeiter bei seinem beruflichen Handeln trifft. Es bleibt jedoch offen, ob die Forderungen aus den konkreten Notwendigkeiten der realen Tätigkeit erwachsen, oder ob es sich um Forderungen eines fremdgesetzten Lehrplans handelt.
Neunter Widerspruch: Die Arbeitsagentur fordert, dass den Mitarbeitern in ihren Lernprozessen eine »sozialpädagogische Betreuung " zuteil wird (Punkt 3.4.). Das kann im Einklang mit dem Prinzip stehen, die Person bei der Verarbeitung von Erfahrungen zu unterstützen, bei denen eine Diskrepanz zwischen den Anforderungen der Aufgabe und ihren persönlichen Fähigkeiten deutlich wird. Es bleibt jedoch offen, ob die Betreuung dient, und bei der die Personen allgemeinverbindliche Curricula zu absolvieren haben.

Zwölfter Widerspruch: Die Arbeitsagentur fordert, dass Betriebspraktika "verbindlicher Teil der Berufsbildung " sind (Punkt 5.2.). Das kann im Einklang mit dem Prinzip stehen, die Lernorte so zu wählen, dass sie die Lernhandlungen ermöglichen, die jeweils weiterführen. Es

\section{»Möglicherweise will sich die Arbeitsagentur langfristig aus der ungeliebten Finanzierung von Einrichtungen der Behindertenbilfe zurückzuziehen"}

primär dazu dienen soll, Lernprozesse zu ermöglichen, deren Inhalte und Verläufe jeweils ausgehandelt werden, oder ob sie »individuell « sicherstellen soll, dass die Person vorgezeichneten Lernprogrammen folgt.

Zehnter Widerspruch: Die Arbeitsagentur fordert, dass die Fähigkeiten der Mitarbeiter " planmäßig « entwickelt werden (Punkt 2.) und dass der Eingliederungsplan das »Eingliederungsziel « benennt (Punkt 3.2.2.). Das kann im Einklang mit dem Prinzip einer arbeitsbezogenen Bildung stehen, bei dem sich das beiläufige Lernen des Mitarbeiters zu einem bewussten, gewollten und systematischen Lernen entwickelt; es bleibt jedoch offen, ob diese Intentionalität des Lernens gefördert werden soll oder die Mitarbeiter fremdgesetzten Zielen und Plänen untergeordnet werden sollen.

Elfter Widerspruch: Die Arbeitsagentur fordert, dass die Werkstätten Rahmenpläne erstellen, die Qualifizierungsinhalte für verschiedene Niveaus beschreiben (Punkt 5.1.); angestrebt wird eine »Ausrichtung an den anerkannten Berufsausbildungen « (Punkt 1.). Das widerspricht dem Prinzip, die Lernplanung individuell anzulegen und eine Lernorganisation $\mathrm{zu}$ vermeiden, die letztlich der Selektion bleibt jedoch offen, ob hier zusätzliche, extramurale Lernmöglichkeiten erschlossen werden sollen oder ob die erwünschte Praxisnähe generell nur »Betrieben und Dienststellen des allgemeinen Arbeitsmarkts « zugebilligt wird (ebd.).

Dreizehnter Widerspruch: Die Arbeitsagentur fordert, dass die Bildung unter anderem » in Form fachpraktischer Unterweisung" stattfinden soll (Punkt 5.1.). Das kann im Einklang mit dem Prinzip stehen, die notwendige Anleitung dadurch zu gewährleisten, dass eine Person im realen Arbeitszusammenhang an den Fähigkeiten anderer teilhat, wie das etwa beim Verhältnis des Auszubildenden zum Meister der Fall ist, wo der Lernende schrittweise in eine konkrete Praxis hineinwächst. Es bleibt jedoch offen, ob dieses partizipative Lernen gefördert werden soll, oder ob an eine Verschulung der beruflichen Bildung gedacht ist.

Vierzehnter Widerspruch: Die Arbeitsagentur fordert, dass "arbeits- und sonderpädagogisch bewährte Lernmodelle und -methoden angewandt " werden (Punkt 5.1.). Das kann im Einklang mit dem Prinzip stehen, Belehrung zu vermeiden und Instruktionen nur problembezogen einzusetzen. Es bleibt jedoch offen, ob die selbsttätige Aneignung als Haupt- 
modus des Lernens unterstützt werden soll, oder ob die Vorstellung besteht, es seien die Werkstätten, die durch didaktische Vorkehrungen Qualifikationen bei ihren Mitarbeitern herstellen und den Erfolg garantieren können.

\section{Resümee}

Wo Widersprüche nicht offen zutage treten, da liegen zumeist Machtverhältnisse und verdeckte Interessen vor. Die Kundenforderungen der Arbeitsagentur haben ihren Schwerpunkt in der politischen Erwartung, dass mit einer konventionellen Art der Berufsbildung die Chancen steigen könnten, behinderte Werkstattmitarbeiter in reguläre Beschäftigungsverhältnisse $\mathrm{zu}$ vermitteln, und dass dies zur Kostendämpfung in der Behindertenhilfe beitragen könnte.

Die Werkstätten werden nicht mehr als Lebensräume für behinderte Bürger gesehen, sondern sie werden nun als Lieferanten von erfolgsorientierten Bildungsmaßnahmen behandelt. Nebenbei suspendiert wird mit diesem Herangehen das Prinzip »erst platzieren, dann qualifizieren «, das sich in der Behindertenhilfe bewährt hat. Mittelfristig soll die Option eröffnet werden, die berufliche Grundqualifizierung behinderter Personen von den Werkstätten zu trennen, um die Förderung auf jene Personen zu beschränken, die am allgemeinen Arbeitsmarkt als vermittelbar erscheinen.
Und man darf getrost annehmen, dass es der Arbeitsagentur langfristig darum geht, sich aus der ungeliebten Finanzierung von Einrichtungen der Behindertenhilfe zurückzuziehen. Vielleicht wäre das für die Behinderten das Beste?

* Bundesagentur für Arbeit: Fachkonzept für Eingangsverfahren und Berufsbildungsbereich in Werkstätten für behinderte Menschen. Nürnberg, 21.06.2010. Internet http://www.arbeitsagentur.de/ zentraler-Content/HEGA-Internet/A03Berufsberatung/Publikation/HEGA-062010-Fachkonzept-WfbM-Anlage.pdf

\section{Das letzte Netz sozialer Sicherung im deutsch- japanischen Vergleich}

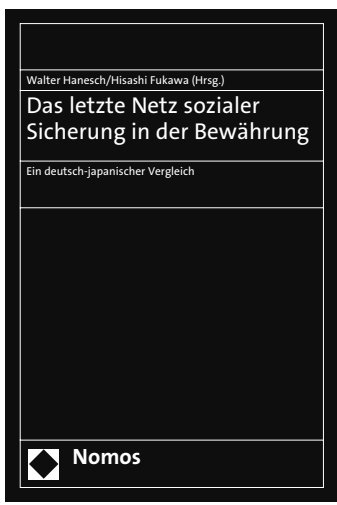

Das letzte Netz sozialer Sicherung in der Bewährung Ein deutsch-japanischer Vergleich Herausgegeben von Prof. Dr. Walter Hanesch und Prof. Dr. Hisashi Fukawa 2011, ca. 250 S., brosch., ca. 49, $-€$ ISBN 978-3-8329-5965-4 Erscheint ca. Oktober 2011 nomos-shop.de/12916

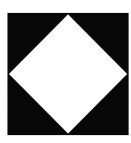
Nomos

\section{Was versteht man eigentlich unter Eingangsverfahren und Berufsbildungsbereich} in Werkstätten für behinderte Menschen?

Der Werkstatt für behinderte Menschen liegt ein Organisationsschema zugrunde, das zwischen Eingangsverfahren, Berufsbildungsbereich und Arbeitsbereich differenziert. Das Eingangsverfahren dauert drei Monate und ist obligatorisch durchzuführen. Es dient der Feststellung, ob die Werkstatt die geeignete berufliche Fördermaßnahme für den behinderten Menschen darstellt oder ob andere Maßnahmen in Betracht kommen. Dem Eingangsverfahren folgt eine in der Regel zweijährige berufliche Qualifizierungsphase im Berufsbildungsbereich der Werkstatt. Der Berufsbildungsbereich gliedert sich in einen Grund- und einen Aufbaukurs von jeweils zwölf Monaten, in denen berufsspezifische Fähigkeiten und Fertigkeiten erlernt werden sollen. Vor allem soll das Selbstwertgefühl der Werkstattbeschäftigten entwickelt und das So- zial- und Arbeitsverhalten gefördert werden. Nach dem Berufsbildungsbereich besteht für Werkstattbeschäftigte die Möglichkeit, in den Arbeitsbereich der Werkstatt für behinderte Menschen zu wechseln.

Günter Mosen

Günter Mosen ist Vorsitzender der Bundesarbeitsgemeinschaft Werkstätten für behinderte Menschen e. V.

Quelle: Deutscher Verein für öffentliche und private Fürsorge e. V. (Hg.): Fachlexikon der sozialen Arbeit. 7. Auflage. Nomos Verlagsgesellschaft, Baden-Baden 2011. Ca. 1.200 Seiten. 44,- Euro. ISBN 978-3-8329-5153-5. S. 973 f. 